\title{
Simulation Framework for Orbit Propagation and Space Trajectory Visualization
}

\author{
Abolfazl Shirazi ${ }^{1}$ \\ Basque Center for Applied Mathematics BCAM, Bilbao, 48009, Spain \\ University of the Basque Country UPV/EHU, Donostia, 20018, Spain \\ Josu Ceberio ${ }^{2}$ \\ Intelligent Systems Group, University of the Basque Country UPV/EHU, Donostia, 20018, Spain \\ Jose A. Lozano ${ }^{3}$ \\ Basque Center for Applied Mathematics BCAM, Bilbao, 48009, Spain \\ University of the Basque Country UPV/EHU, Donostia, 20018, Spain
}

\begin{abstract}
In this paper, an interactive tool for simulation of satellites dynamics and autonomous spacecraft guidance is presented. Different geopotential models for orbit propagation of Earth-orbiting satellites are provided, which consider Earth's gravitational field with various accuracies. The presented software is a $3 \mathrm{D}$ visualization platform for space orbit simulation with analytical capabilities through various modules. Taking advantage of a graphical user interface, it can evaluate, analyze and illustrate the motion of satellite based on different orbit propagation schemes. Several cases of satellites and autonomous spacecraft in the space rendezvous mission are simulated regarding different propagation models to demonstrate the performance of the application in space mission analysis, ground track visualization and trajectory optimization. Results are validated by comparing with other state-of-the-art tools, such as AGI System Toolkit (STK).
\end{abstract}

Keywords: Orbit Simulation, Software Development, Visualization, Modeling, Spacecraft Dynamics

\section{Introduction}

Modeling and simulation of autonomous spacecraft have made momentous strides in recent years, and the space industry, and other aerospace professions are on the verge of being able to use computing power. The aim of this usage is to simulate reality for all kinds of applications in space engineering such as autonomy of nanosatellites [1], flight simulation [2], space object registration [3], and orbit propagation [4]. In space engineering, rapid simulation of space orbits and trajectories is essential in different aspects of space engineering including trajectory optimization [5], orbit transfers, orbit determination and attitude control, and gravitational modeling. Representation of the dynamical states of spacecraft while moving in an orbit is non-trivial for Earth orbiting satellites. Although preliminary analysis of satellite space orbits can be done without extensive simulation, interactive environments in simulation frameworks allow researchers to design space missions in a broad view that are difficult, expensive, or time consuming to deal with. Programming languages such as Java have been well used in different aspects of space engineering [6].

\footnotetext{
${ }^{1}$ Corresponding author, ashirazi@bcamath.org

2josu.ceberio@ehu.eus

${ }^{3}$ IEEE Fellow, jlozano@bcamath.org
} 
However, the amount of simulation frameworks in space engineering with MATLAB has increased in recent years. This is due to the fact that the capability of MATLAB to manage matrices is dominant and the vast number of libraries and toolboxes are available in this programming language. In recent years, several applications for orbit simulation have been developed including various MATLAB-based toolboxes. The simulation package of AGI, named Systems Tool Kit (STK) [7] is a reputed analytical framework. It has the capability of simulating dynamic environment and scheduling of events within a space mission and has a great contribution in the space engineering community. It also has the capability of integrating with MATLAB, which makes it more flexible for different types of users. The General Mission Analysis Tool (GMAT) [8] is another space mission analysis software package developed by NASA. The MATLAB interface in this software supports connections to the MATLAB environment, letting GMAT to run MATLAB scripts simultaneously.

In addition to the applications with MATLAB integration capabilities, some MATLAB toolboxes have also been developed in recent years for space orbit simulation and design. In 2015, Carrara [9] presented PROPAT, a satellite attitude and orbit analysis tool developed in MATLAB. Although the system's attitude simulation is well-developed based on attitude kinematics and dynamics, the orbit simulation process on the other hand is limited. The propagation model is based on a solution that is achieved analytically, called as Brower model [10]. Based on this model, only three orbital elements including right ascension of ascending node, argument of perigee and mean anomaly are affected by the orbital perturbations. The toolbox SPACSSIM has been introduced in [11] as another software for orbit propagation and attitude control. There are other toolboxes in this matter as well [12]. However, there is a need for a user friendly MATLAB-based application with interactive visualization capability, in which the design, simulation and trajectory optimization within the preliminary design of space systems can be tackled.

In this paper, a simulation platform, called HOMA, is presented to simulate space trajectories around any celestial masses along with orbit analysis. The toolbox includes an orbit propagator, linked to a visualization platform with a user-friendly interface. Various orbital perturbations can be considered including Earth atmospheric drag, Earth harmonic gravity field, solar perturbations and the perturbations of other planets. Three orbit propagators are included regarding these perturbations such as two-body propagator, Simplified General Propagator (SGP4) and High Precision Orbit Propagator (HPOP). Several ordinary differential equation (ODE) solvers are considered to be used within the orbit propagation. Moreover, simulation of the satellite ground track is also included along with several scripts to perform coordinate transformation and calculation of ephemeris.

HOMA toolbox has been developed in two versions, including a MATLAB-based version and an online version [13] for space orbit simulation and analysis. Compared with other similar available MATLAB toolboxes, the MATLAB-based version benefits from a user friendly GUI and a 3D visualization platform, where the user can interact with the space orbit environment while simulating space trajectories. Rapid and instant computation and result generation make the application very practical in the preliminary design of space orbits. This paper presents the description of the HOMA framework, as well as some simulations performed in this application. Results are validated with similar software and toolboxes.

This paper is organized as follows. An overview of the space orbit propagation process in HOMA is provided and the main architecture of the application is described in Section 2. Following the structure of space orbit simulation, different orbit propagation schemes in HOMA are presented in Section 3. The environment of HOMA is introduced in Section 4, in which the different elements of the application interface are presented. Several examples of space orbits are simulated with the application and the results are provided in Section 5. Besides, a multi-impulse orbit transfer problem is simulated and analyzed. This section also includes the comparison of the results with those of state-of-art toolboxes. Finally, the conclusions are provided in Section 6. 


\section{Overall Application Scope}

Simulation of space orbit trajectories involves two elements. The first is the mathematical model of the spacecraft, which describes the dynamics of the system's motion, and the second is the propagation scheme, in which the simulation process marches through the specified time. Following these bases, the overall architecture of HOMA for simulation of space orbits is illustrated in Fig. 1.

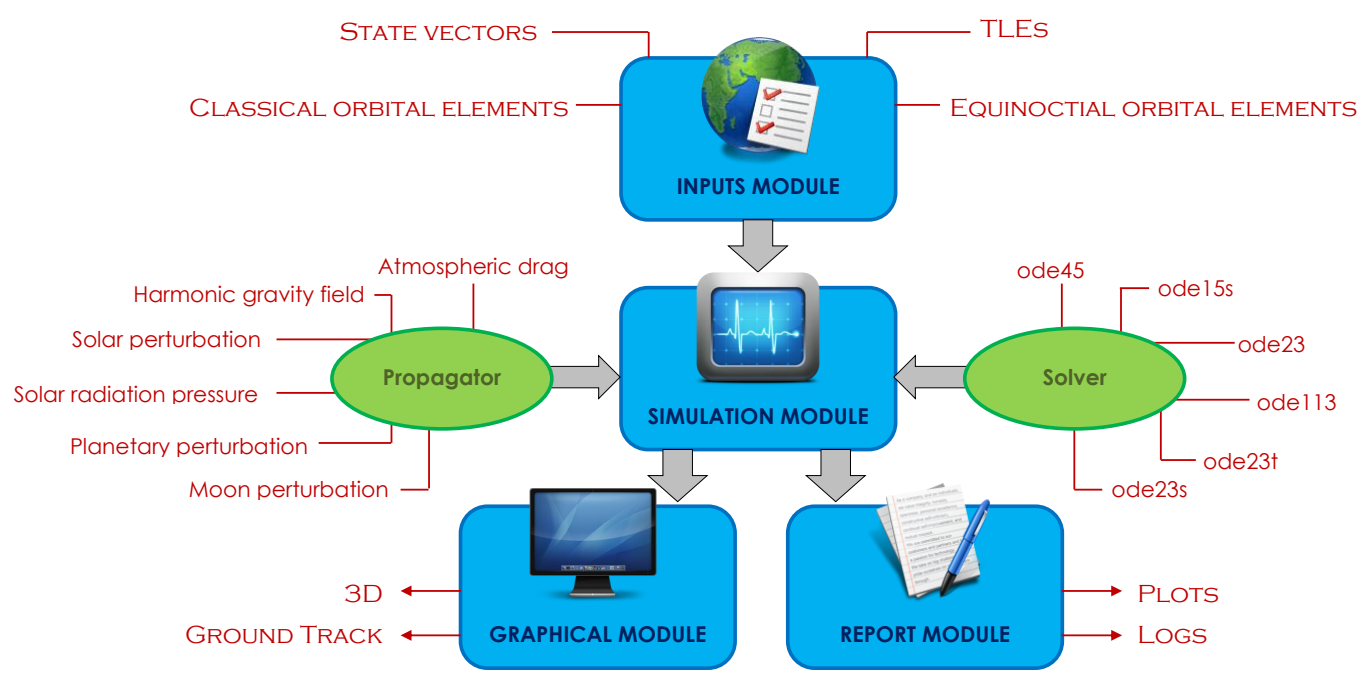

Figure 1: The main architecture of the simulation platform

As shown, the application has an input module in which the inputs of the simulation are defined. Generally the initial state of the spacecraft can be defined in several ways in orbital mechanics. The state vector describes the position and the velocity of the spacecraft, which can also be converted to other forms. There are two other possible representations supported in the software, including classical orbital elements and equinoctial orbital elements [14]. The difference between these representations is their practicality in space missions [15]. In addition to these representations, the well-known two-line-elements sets (TLEs) [16] can also be imported in the software in order to define the state of satellites.

The simulation module includes a propagator and a solver. In the first step of simulating the space vehicle in a space orbit, the dynamics of the spacecraft needs to be mathematically modeled. The motion of the space vehicle in orbit is defined with a set of ordinary differential equations. These equations express a trajectory in terms of position and velocity of the space vehicle as time-dependence variables. Such mathematical model relies on having two point masses and the mutual gravitation force between these masses is taken into account [17]. In this type of mathematical model, it is assumed that the mass of the space vehicle is much smaller and negligible in comparison to central body. Having this assumption, the gravitational effect due to the mass of the space vehicle is ignored.

Considering this set of ordinary differential equations, orbit propagators are utilized to find the propagated position and velocity of the space vehicle. Various perturbations, faced by the satellite in space, are also incorporated to portray the actual behavior of the spacecraft throughout its space mission. Details regarding the orbit propagation in HOMA, such as SGP4 and HPOP propagations, are provided in the following section.

Regarding the propagation model (except for SGP4), a solver is needed to deal with the system of ODEs in several time steps. The solver part offers different ODE solvers for this where the user can specify which solver should be implemented. The choice of the solver makes it possible for the user to analyze and compare different integration methods with each other and evaluate their accuracy and computation time. It is worthy to note that the computation time varies by the propagation model when simulating several trajectories of satellites. Since the propagation process depends on the complexity of system dynamics 
and the stiffness of the equations, the user needs to take a balance between the expected accuracy and the dedicated time for simulation.

After the simulation process, results can be reported and illustrated in different forms including 2D plots of state variables, variation of orbital elements and satellite ground track. $3 \mathrm{D}$ renders of satellite motion can also be generated with different rendering options and settings as images or animations.

\section{Spacecraft Dynamics}

The process of orbit propagation for an autonomous spacecraft in HOMA is based on simulating the orbit for an extended time period using the dynamic equations of motion, models of environmental forces, torques and other physical parameters. In any space mission analysis, prediction of the orbits of satellites is an essential part and it, directly or indirectly, has impacts on the satellite's power system, attitude control, thermal design and other systems. The main factors affecting the orbit of a satellite are the non-spherical geometry of Earth, atmospheric drag, perturbed effects from the gravitational pull of the Sun and other planets, electromagnetic forces, radiation pressures and so forth. As stated, the software considers a number of propagators available including two-body, SGP4 and HPOP. The mathematical representation for the dynamics of the space vehicle regarding the aforementioned assumptions can be defined as the non-Keplerian two-body problem equation, [14]:

$$
\vec{r}=-\frac{\mu}{r^{3}} \vec{r}+\vec{\gamma}
$$

This representation is defined based on the inertial coordinate system, where $\vec{r}$ is the position of the space vehicle $(r=|\vec{r}|)$, respect to the inertial coordinate frame, $\mu$ is a constant, describing the central mass gravitational property, and $\vec{\gamma}$ is the acceleration, which affects the space vehicle motion because of orbital perturbations.

\subsection{Two-body Propagation}

The simple and traditional space orbit simulation method is the two-body orbit propagation. Considering $\vec{\gamma}=0$ in Eq. 1, the two-body model will provide a rough idea of a spacecraft's orbit. There are two ways to simulate the spacecraft motion in this model. The first way is by solving the ODE set of equations in Eq. 1 using iterative methods. Different ODE solvers can be utilized in this case. The second way is to simulate the satellite motion and velocity in the form of orbital elements. Since the perturbation is assumed to be zero, the motion will be on a Keplerian trajectory and the true anomaly domain will be considered instead of time for simulation. Conversion of time to true anomaly for different conic sections can be found in [18].

\subsection{SGP4 Propagator}

The SGP4 model simulates the motion of the space vehicle with a few considerations. In this model the effect of perturbations has been taken into account while computing the state vectors. The perturbations due to the shape of the Earth, the drag force due to atmosphere, Sun radiation, and the acceleration due to the gravity of other giant masses such as the Sun and Moon are involved in this model. The two-line element sets from United States Space Command are utilized for orbit propagation with SGP4 model. The general scheme of SGP4 propagator is presented in Fig. 2 based on its available package [19]. This flowchart does not provide the details in this propagator, but it does provide an overview of the process.

The propagator includes two main steps which are the initialization and the main loop. It is coupled with many callbacks and scripts. However, the key-element functions are limited. The structure containing all the SGP4 satellite information is stored in SatRec. This variable will be read and updated throughout the whole process. After setting the 


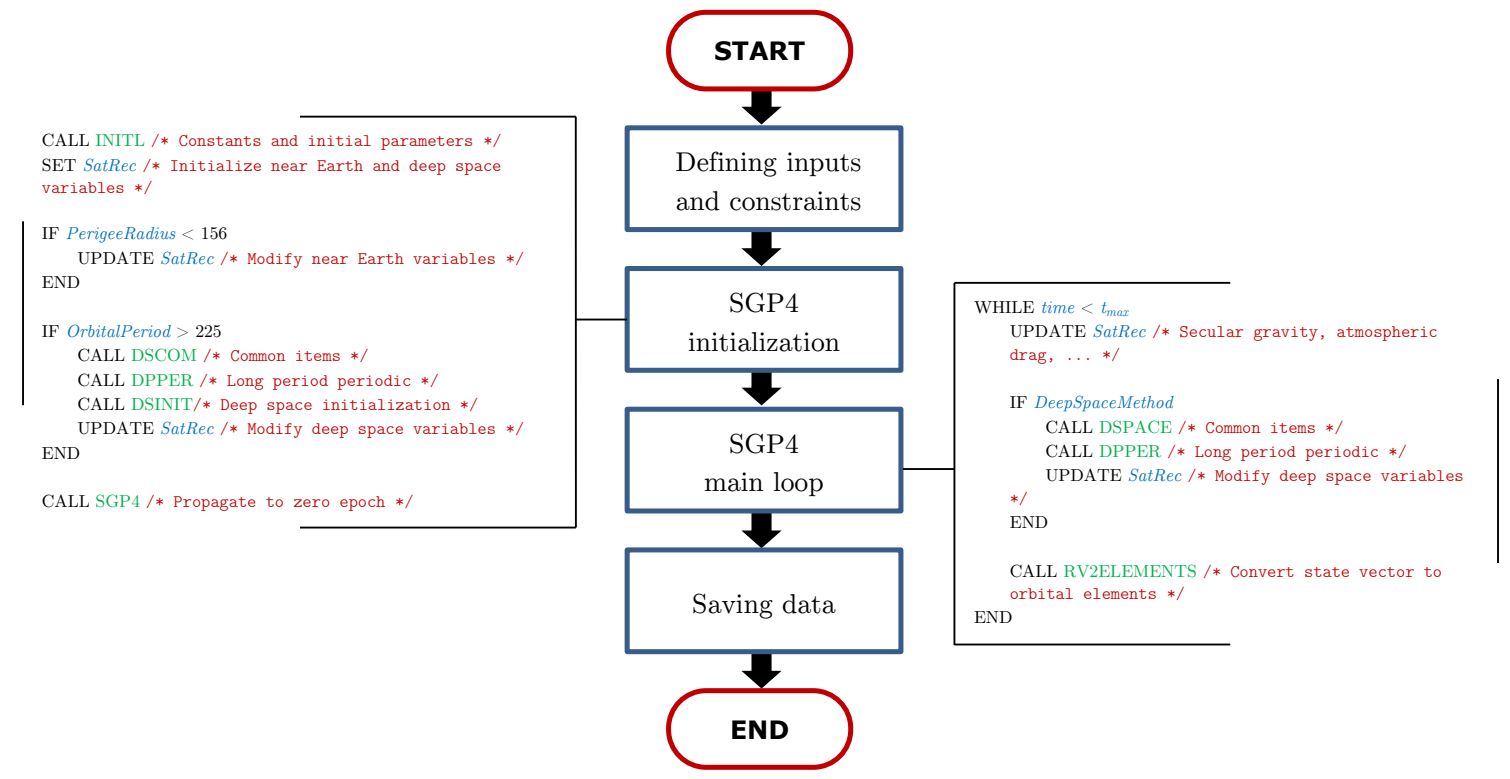

Figure 2: Flowchart of SGP4 propagator

input data, initialization will be handled first. Then a time loop updates the propagator structure and calculates the state vectors. Finally, the results will be generated according to the desired form.

\subsubsection{Initialization}

The SGP4 propagation includes several constant variables, which do not depend on time. Therefore, the propagation process starts with calculating these terms (INITL). This step is outlined as follows [20, 21]:

$$
\begin{aligned}
a_{1} & =\left(\frac{k_{e}}{n_{0}}\right)^{\frac{2}{3}} \\
\delta_{1} & =\frac{3}{2} \frac{k_{2}}{a_{1}^{2}} \frac{\left(3 \cos ^{2} i_{0}-1\right)}{\left(1-e_{0}^{2}\right)^{\frac{3}{2}}} \\
a_{2} & =a_{1}\left(1-\frac{1}{3} \delta_{1}-\delta_{1}^{2}-\frac{134}{81} \delta_{1}^{3}\right) \\
\delta_{0} & =\frac{3}{2} \frac{k_{2}}{a_{2}^{2}} \frac{\left(3 \cos ^{2} i_{0}-1\right)}{\left(1-e_{0}^{2}\right)^{\frac{3}{2}}} \\
n_{0}{ }^{\prime \prime} & =\frac{n_{0}}{1+\delta_{0}} \\
a_{0}{ }^{\prime \prime} & =\left(\frac{k_{e}}{n_{0}{ }^{\prime \prime}}\right)^{\frac{2}{3}}
\end{aligned}
$$

where $k_{2}=\frac{1}{2} J_{2} a_{E}^{2}$ (units of Earth radii), $J_{2}=1.082616 \times 10^{-3}, k_{e}=\sqrt{G M}=0.0743669161$, $G$ is the universal gravitational constant, $M$ is the mass of Earth, and $a_{E}$ is the equatorial radius of Earth.

Acceleration due to atmosphere drag is the next variable that is considered in initialization. The mathematical model of this term is based on a density function from power law as in [22].

$$
\rho=\rho_{0} \frac{\left(q_{0}-s\right)^{4}}{(r-s)^{4}}
$$


In this equation, $r$ represents the distance between the space vehicle and the Earth's center, $q_{0}$ and $s$ are the parameters for the density function. The value of $q_{0}$ is $120 \mathrm{~km}$ plus the radius of Earth and the value of $s$ is the height of the spacecraft at perigee. In this model, if the perigee altitude becomes less than $156 \mathrm{~km}$, the value of some of the parameters will be altered within the initialization. So the SatRec structure will be updated accordingly:

$$
\begin{aligned}
\theta= & \cos \left(i_{0}\right) \\
\xi= & \frac{1}{a_{0}-s} \\
\beta_{0}= & \left(1-e_{0}^{2}\right)^{\frac{1}{2}} \\
\eta= & a_{0} e_{0} \xi \\
C_{2}= & \left(q_{0}-s\right)^{4} \xi^{4} n_{0}\left(1-\eta^{2}\right)^{-\frac{7}{2}} \times\left[a_{0}\left(1+\frac{3}{2} \eta^{2}+4 e_{0} \eta+e_{0} \eta^{3}\right)+\right. \\
& \left.\frac{3}{2} \frac{k_{2} \xi}{\left(1-\eta^{2}\right)}\left(-\frac{1}{2}+\frac{3}{2} \theta^{2}\right)\left(8+24 \eta^{2}+3 \eta^{4}\right)\right] \\
C_{1}= & B \times C_{2} \\
C_{3}= & \frac{\left(q_{0}-s\right)^{4} \xi^{5} A_{(3,0)} n_{0} a_{E} \sin i_{0}}{k_{2} e_{0}} \\
C_{4}= & 2 n_{0}\left(q_{0}-s\right)^{4} \xi^{4} a_{0} \beta_{0}^{2}\left(1-\eta^{2}\right)^{-\frac{7}{2}}\left\{\left[2 \eta\left(1+e_{0} \eta\right)+\frac{1}{2} e_{0}+\frac{1}{2} \eta^{3}\right]-\right. \\
& \frac{2 k_{2} \xi}{a_{0}\left(1-\eta^{2}\right)}\left[3\left(1-3 \theta^{2}\right)\left(1+\frac{3}{2} \eta^{2}-2 e_{0} \eta-\frac{1}{2} e_{0} \eta^{3}\right)+\right. \\
& \left.\left.\frac{3}{4}\left(1-\theta^{2}\right)\left(2 \eta^{2}-e_{0} \eta-e_{0} \eta^{3}\right) \cos \left(2 \omega_{0}\right)\right]\right\} \\
C_{5}= & 2\left(q_{0}-s\right)^{4} \xi^{4} a_{0} \beta_{0}^{2}\left(1-\eta^{2}\right)^{-\frac{7}{2}}\left[1+\frac{11}{4} \eta\left(\eta+e_{0}\right)+e_{0} \eta^{3}\right] \\
D_{2}= & 4 a_{0} \xi C_{1}^{2} \\
D_{3}= & \frac{4}{3} a_{0} \xi^{2}\left(17 a_{0}+s\right) C_{1}^{3} \\
D_{4}= & \frac{2}{3} a_{0}^{2} \xi^{3}\left(221 a_{0}+31 s\right) C_{1}^{4}
\end{aligned}
$$

where $A_{3,0}=-J_{3} a_{E}{ }^{3}$, and $J_{3}=-0.253881 \times 10^{-5}$. Zonal harmonics of the Earth are considered in initialization as:

$$
\begin{aligned}
\dot{M}= & {\left[\frac{3 k_{2}\left(-1+3 \theta^{2}\right)}{2 a_{0}{ }^{2} \beta_{0}{ }^{3}}+\frac{3 k_{2}{ }^{2}\left(13-78 \theta^{2}+137 \theta^{4}\right)}{16 a_{0}{ }^{4} \beta_{0}{ }^{7}}\right] n_{0} } \\
\dot{\omega}= & {\left[-\frac{3 k_{2}\left(1-5 \theta^{2}\right)}{2 a_{0}{ }^{2} \beta_{0}{ }^{4}}+\frac{3 k_{2}{ }^{2}\left(7-114 \theta^{2}+395 \theta^{4}\right)}{16 a_{0}{ }^{4} \beta_{0}{ }^{8}}+\right.} \\
& \left.\frac{5 k_{4}\left(3-36 \theta^{2}+49 \theta^{4}\right)}{4 a_{0}{ }^{4} \beta_{0}{ }^{8}}\right] n_{0} \\
\dot{\Omega}= & {\left[\frac{3 k_{2} \theta}{a_{0}{ }^{2} \beta_{0}{ }^{4}}+\frac{3 k_{2}{ }^{2}\left(4 \theta^{2}-19 \theta^{3}\right)}{2 a_{0}{ }^{4} \beta_{0}{ }^{8}}+\frac{5 k_{4} \theta\left(3-7 \theta^{2}\right)}{2 a_{0}{ }^{4} \beta_{0}{ }^{8}}\right] n_{0} }
\end{aligned}
$$

where $k_{4}=-\frac{3}{8} J_{4} a_{E}{ }^{4}$, and $J_{4}=-1.65597 \times 10^{-6}$.

The secular coefficient and the long-period coefficient for solar and lunar gravity are also initialized as the mentioned variables are calculated. This computation includes some 
additional terms if the orbital period of the space vehicle is greater or equal to 225 minutes (DSCOM, DPPER, DSINIT). Details are provided in [23]. Lastly, when all of the initial variables are computed, the orbit is propagated once to initialize the states at epoch time.

\subsubsection{Update and Iteration}

After the initialization, the simulation goes through a loop in time domain in which the spacecraft trajectory is propagated with the constant variables calculated previously. The updating steps considers short-period and long-period effects of solar and lunar gravity, resonance effects of Earth gravity, and atmospheric drag effects.

Considering these updates, propagation yields the updated parameters in SatRec in each iteration. Then, the vectors representing the unit orientations are computed and the state vectors will be obtained. Knowing the state vectors, the rest of the orbital characteristics can be calculated accordingly (RV2ELEMENTS) as $r_{k}, \dot{r}_{k}, r \dot{f}_{k}, u_{k}, i_{k}$ and $\Omega_{k}$ in each iteration. Unit orientation vectors, as $\vec{U}$ and $\vec{V}$ are determined as:

$$
\begin{aligned}
& \vec{U}=M \sin u_{k}+N \cos u_{k} \\
& \vec{V}=M \cos u_{k}-N \sin u_{k}
\end{aligned}
$$

where

$$
\begin{aligned}
& \vec{M}=\left[\begin{array}{c}
-\sin \Omega_{k} \cos i_{k} \\
\cos \Omega_{k} \cos i_{k} \\
\sin i_{k}
\end{array}\right] \\
& \vec{N}=\left[\begin{array}{c}
\cos \Omega_{k} \\
\sin \Omega_{k} \\
0
\end{array}\right]
\end{aligned}
$$

Then, the position and velocity vectors are calculated by:

$$
\begin{aligned}
& \vec{r}=r_{k} \vec{U} \\
& \vec{v}=\dot{r}_{k} \vec{U}+r \dot{f}_{k} \vec{V}
\end{aligned}
$$

The simplified general perturbations consider secular effects of $J 2, J 4$, and long-periodic effects of $J 3$, and short-periodic effects of $J 2$, along with atmospheric drag. This propagation method is very popular due to its widespread application for various kinds of missions. More detailed description of the SGP4 model as applied for the generation of NORAD 2-line elements is provided in [19].

\subsection{High-Precision Orbit Propagator (HPOP)}

The HPOP model consists of a propagation process in which the general orbital perturbations along with the gravitational forces due to other planets (N-body problem) are taken into account. As a result, this model provide more accurate prediction in comparison to other propagation models and provide a base for modeling much more complicated trajectories.

As in HPOP, the equations of acceleration in Eq. 1 of a space vehicle are computed in the inertial reference frame as:

$$
\vec{\gamma}=\vec{\gamma}_{g}+\vec{\gamma}_{n g}
$$


where $\vec{\gamma}_{g}$ is the sum of the accelerations due to gravitational forces, affecting the spacecraft motion other than the typical term for Earth-gravity $\left(-\mu / r^{3} \vec{r}\right)$ in Eq. 1, and $\vec{\gamma}_{n g}$ is the sum of the non-gravitational forces, which are acted on the space vehicle surface areas.

Obviously, the main difference between this model and the two-body model is that the perturbation term is not assumed to be zero $(\vec{\gamma} \neq 0)$. The main challenge in HPOP is the precise calculation of different perturbation terms. For the sake of brevity, details of these terms are omitted here and the reader is urged to refer to the references in the following sections.

The first group of terms in HPOP is the gravitational perturbations. The acceleration due to gravitational forces in this term can be expressed as follows.

$$
\vec{\gamma}_{g}=\vec{\gamma}_{g e o}+\vec{\gamma}_{s t}+\vec{\gamma}_{o t}+\vec{\gamma}_{r d}+\vec{\gamma}_{n}+\vec{\gamma}_{r e l}
$$

where $\vec{\gamma}_{g e o}$ is the term describing the orbital perturbations caused by the Earth's geopotential, $\vec{\gamma}_{s t}$ is the perturbation caused by solid Earth tides, $\vec{\gamma}_{o t}$ is the perturbation caused by the ocean tides, $\vec{\gamma}_{r d}$ is the effect of rotational deformation, $\vec{\gamma}_{n}$ is the gravitational effect of other giant masses such as Sun, Moon and planets, and $\vec{\gamma}_{r e l}$ is the perturbations caused by the general relativity.

The gradient of the potential function, $U$, that satisfies the Laplace equation, $\nabla^{2} U=0$, represents the Earth gravitational attraction. The resulting perturbation acceleration can be specified as follows:

$$
\begin{aligned}
\nabla U & =\nabla\left(U_{s}+\Delta U_{s t}+\Delta U_{o t}+\Delta U_{r d}\right) \\
& =\vec{\gamma}_{g e o}+\vec{\gamma}_{s t}+\vec{\gamma}_{o t}+\vec{\gamma}_{r d}
\end{aligned}
$$

In this equation, $U_{s}$ denotes the potential caused by solid-body mass distribution. The effect of solid-body tides is represented by $\Delta U_{s t}, \Delta U_{o t}$ denotes the effect of potential changes due to the ocean tides, and $\Delta U_{r d}$ is the effect of the rotational deformations.

As for $U_{s}$, spherical harmonic expansion is usually utilized to express this variable, with respect to the body-fixed reference frame [24]. Also, since a non-rigid elastic body is the real formation of the Earth, its mass distribution varies with a non-uniform pattern. The solid Earth tides that affect the variation of $\left(\Delta U_{s d}\right)$ are commonly expressed by external geopotentials as described in [25]. Moreover, details regarding the computation of oceanic tidal perturbations $\left(\Delta U_{o t}\right)$ and rotational deformation $\left(\Delta U_{r d}\right)$ can be found in [26] and [27] respectively.

Acceleration due to the gravity of other planets is determined based on point mass approximations with high accuracy. The $N$-body accelerations with respect to the geocentric inertial coordinate frame, can be calculated as the following:

$$
\vec{\gamma}_{n}=\sum_{i=1}^{N} G M_{i}\left[\frac{\vec{r}_{i}}{r_{i}{ }^{3}}-\frac{\vec{\Delta}_{i}}{\Delta_{i}{ }^{3}}\right]
$$

in this equation, universal gravitational constant is represented by $G, M_{i}$ denotes the mass of the $i$-th body-mass, $\vec{r}_{i}$ represents the position vector of the $i$-th body-mass and $\vec{\Delta}_{i}$ is the position vector of the i-th body-mass relative to the space vehicle. Details regarding the calculation of these parameters can be found in [28]. Also, the mathematical model of relativistic perturbations for Earth-orbiting spacecraft is discussed in details in [29] and [30].

Finally, the accelerations due to non-gravitational forces that affects the motion of the spacecraft are the second group of terms and can be expressed as the following.

$$
\vec{\gamma}_{n g}=\vec{\gamma}_{\text {drag }}+\vec{\gamma}_{\text {solar }}+\vec{\gamma}_{\text {Earth }}+\vec{\gamma}_{\text {thermal }}
$$


where $\vec{\gamma}_{d r a g}$ is the acceleration due to the atmospheric drag [31], $\vec{\gamma}_{\text {solar }}$ is the effect of solar radiation pressure [32], $\vec{\gamma}_{\text {Earth }}$ is the perturbations because of the radiation pressure of Earth [33], and $\vec{\gamma}_{\text {thermal }}$ is the perturbations owing to the thermal radiation [34]. All of these parameters are surface-dependent and therefore their calculations depend on the shape and orientation of the space vehicle.

\section{Application Environment}

Within HOMA, the user can design and construct trajectories that reflect the space mission orbit regarding the desired orbit propagation schemes as described previously. The user interface of the application is shown in Fig. 3.

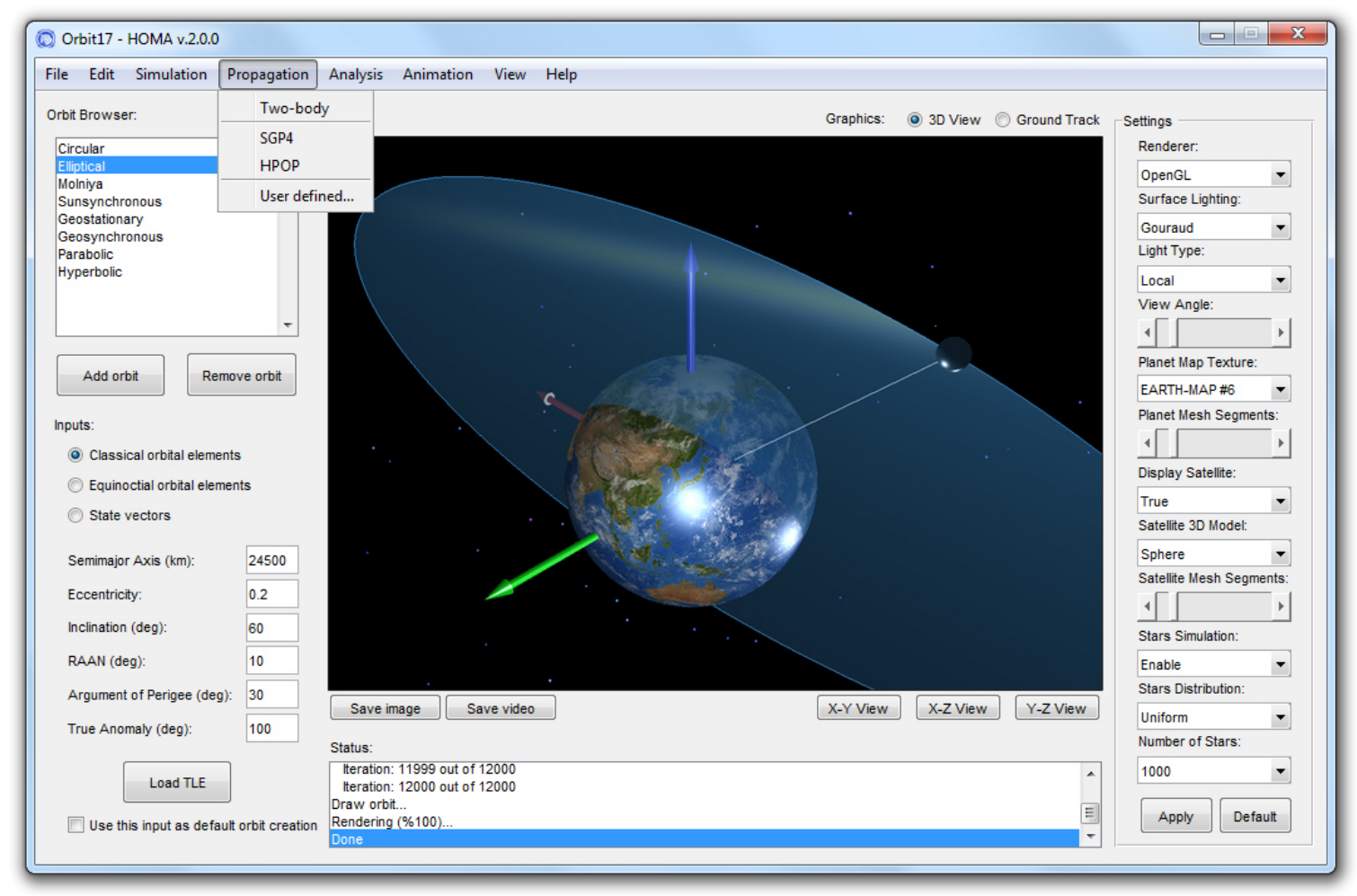

Figure 3: Main GUI of the HOMA toolbox

The user can specify state vectors, orbital elements or load TLEs to define the orbits. These values are then propagated in the simulation environment incorporating different perturbations according to the type of propagator. It is also possible for the user to use their own propagator within the simulation. This process may involve numerical iterations.

As Fig. 3 shows, the orbit browser has the ability to save and keep the highly used and most frequent types of orbits which are simulated by the user. Regardless of the propagator or the solver, the application instantly converts the state vectors and orbital elements to each other. A module is also available for manually entering TLEs.

The graphic frame can be switched between two types of visualization: $3 \mathrm{D}$ view and ground track. In the 3D view frame, the orbit alongside the central space mass is illustrated and it enables the user to perform a number of viewing operations interactively. The camera position can be set as free or fixed with respect to a specific point relative to the spacecraft. In the ground track frame, the ground track of the satellite is shown. Different numbers of revolutions can be specified by the user for plotting the ground track as well as various epoch times for simulation. Illustrations can be saved as high quality images or animation files while the satellite is in motion within its orbit.

The interface also has a status bar, displaying the current status of operations in the application. It is also possible to change the verbose style and save the status report 
accordingly. The panel for visualization settings includes several options for controlling the graphic frame. These options include the renderer type, lighting options, 3D segments control, planet textures, display options, settings for simulating stars, etc.

\section{Simulation}

In this section, several space orbit instances are simulated and the results are presented to demonstrate the performance of the application. First, the simulation capabilities of the software are demonstrated via some orbit analysis. Variation of state vectors, anomalies, and visualization of orbits have been taken into account in some space orbit examples. Second, the simulation of ground track is taken into consideration. Different geosynchronous orbits are simulated and the results are evaluated regarding the differences in orbital elements. Then, a trajectory optimization problem for long-range space rendezvous is analyzed and simulated to demonstrate the practicality of the proposed application [35]. Finally, comparisons are illustrated between the results from HOMA and two other space orbit simulation software packages including PROPAT and STK.

\subsection{Orbit Analysis}

Consider a spacecraft traveling in an orbit with semi-major axis of $14000 \mathrm{~km}$ and eccentricity of 0.5 , inclined by $60^{\circ}$ with argument of perigee of $10^{\circ}$ and right ascension of ascending node of $80^{\circ}$. The position of the spacecraft is considered to be at a true anomaly of $170^{\circ}$. Visualization of this orbit based on two-body propagation with ode 45 solver is illustrated in Fig. 4, relative to inertial frame.

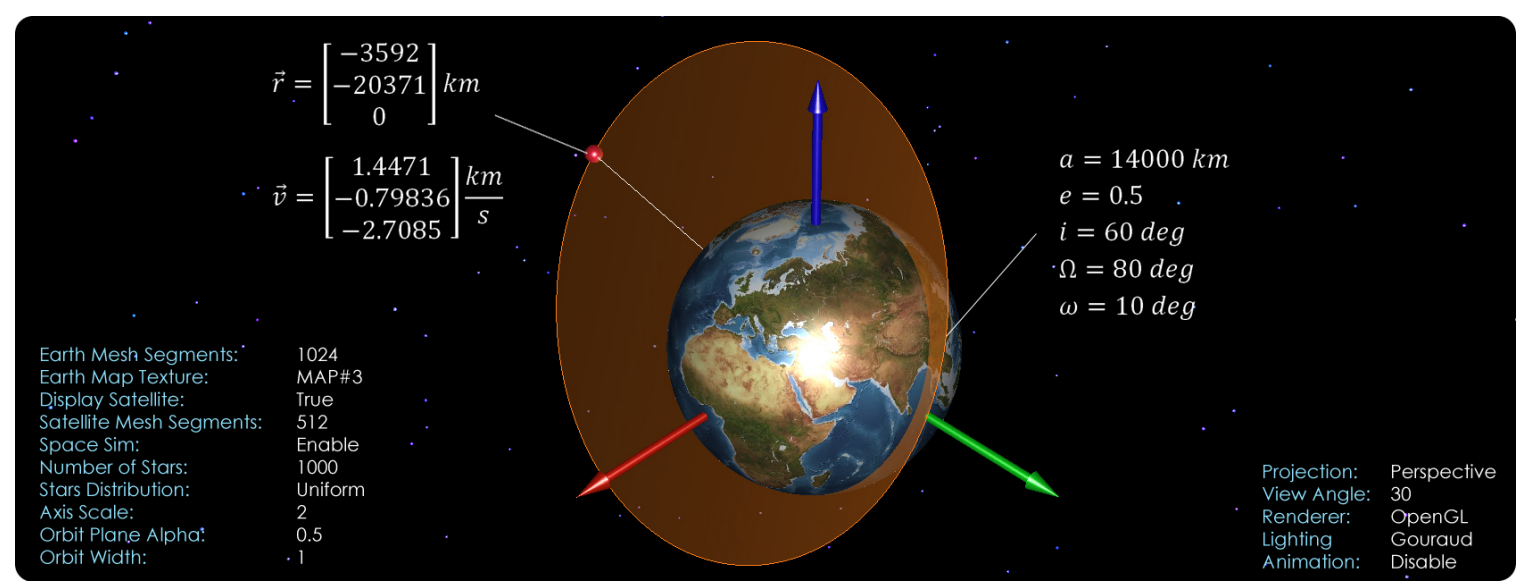

Figure 4: $3 \mathrm{D}$ visualization of a space orbit

As shown, the instant conversion of the orbital elements is performed within the application. This conversion renders the state vectors as $\vec{r}=[-3592 ;-20371 ; 0] \mathrm{km}$ and $\vec{v}=[1.4471 ;-0.79836 ;-2.7085] \mathrm{km} / \mathrm{s}$. Some of the rendering options are also displayed in the rendered scene. As time progresses, no periodic behavior is observed with the orbital elements, but it can be observed with the state vectors since the two-body model is employed. These variations are depicted in Fig. 5 and Fig. 6.

In these figures, the variations of radius and velocity vectors are illustrated as functions of true anomaly. For the shown space mission, one revolution with the orbit corresponds to the total time of 16486 seconds. While using the two-body propagation scheme, the analytical solution of the equation of relative motion is available. However, the simulation is always performed numerically in HOMA, unless specified by the user for special cases.

As another example, one shot of the ballistic view of a satellite moving in an elliptical orbit with perigee radius of $7000 \mathrm{~km}$ and apogee radius of $40000 \mathrm{~km}$ is illustrated in Fig. 7. 


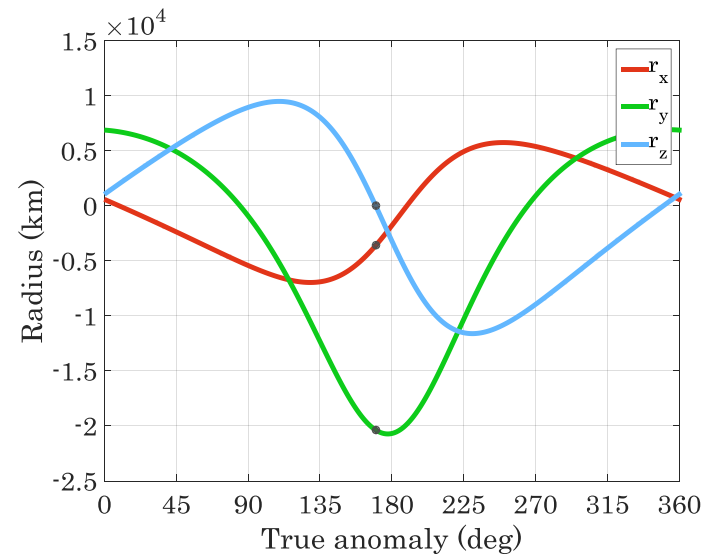

Figure 5: Position $(|\vec{r}|=20685 \mathrm{~km})$

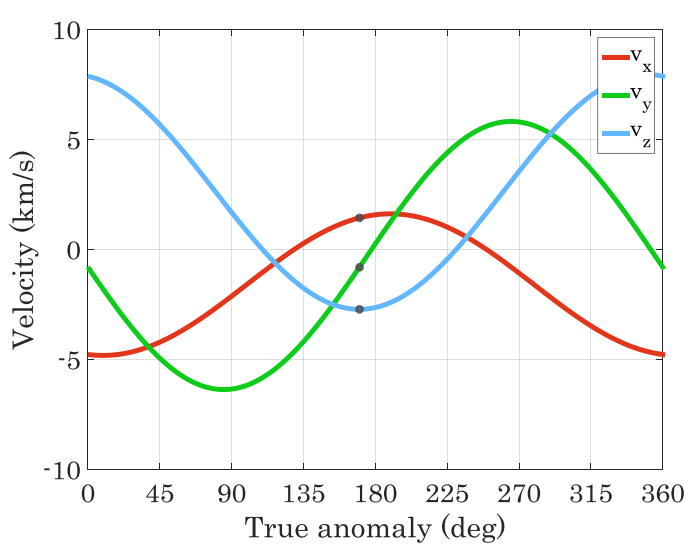

Figure 6: Velocity $(|\vec{v}|=3.1729 \mathrm{~km} / \mathrm{s})$

As shown, it is possible to give different representations of satellite and orbit in 3D visualization frame. Within the simulation of the spacecraft motion in an elliptical space orbit, one can not picture mean anomaly as a physical element of the motion as the eccentric anomaly; rather it is related to time. However, eccentric and mean anomalies can be calculated as functions of each other. Mean, eccentric, and true anomaly are all equal to zero at perigee. Likewise, all three elements are equal to $180^{\circ}$ at apogee. These variations are depicted in Fig. 8 for the an orbit with a given eccentricity.

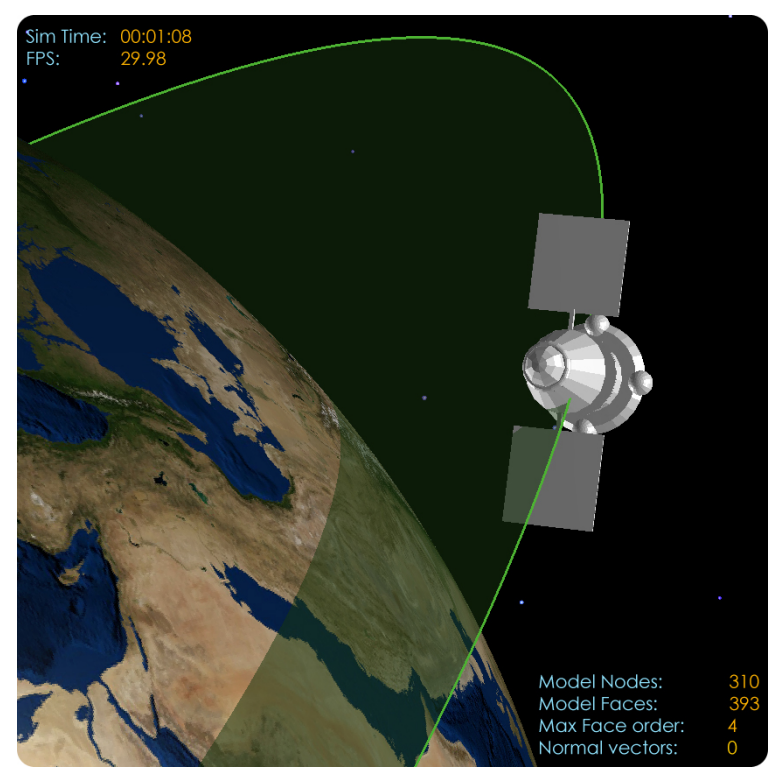

Figure 7: Orbit visualization (ballistic view)

Fig. 8 also represents the flight path angle. This angle is simply the one that the local horizon makes with the velocity vector $\vec{v}$ which is normal to the position vector. The sign of this angle is positive as the space vehicle travels away from perigee and is negative as the space vehicle is approaching the perigee point. This angle plays an important role while calculating the required velocity increment within an orbit transfer. When a $\Delta v$ is computed for an impulsive orbit transfer at a point that is not on the apse line, including the variations of direction is an important matter as well as the magnitude of the velocity vector. The difference in the magnitude of the two vectors identified the change in the velocity, and the difference in the flight path angles shows the change in the direction. 


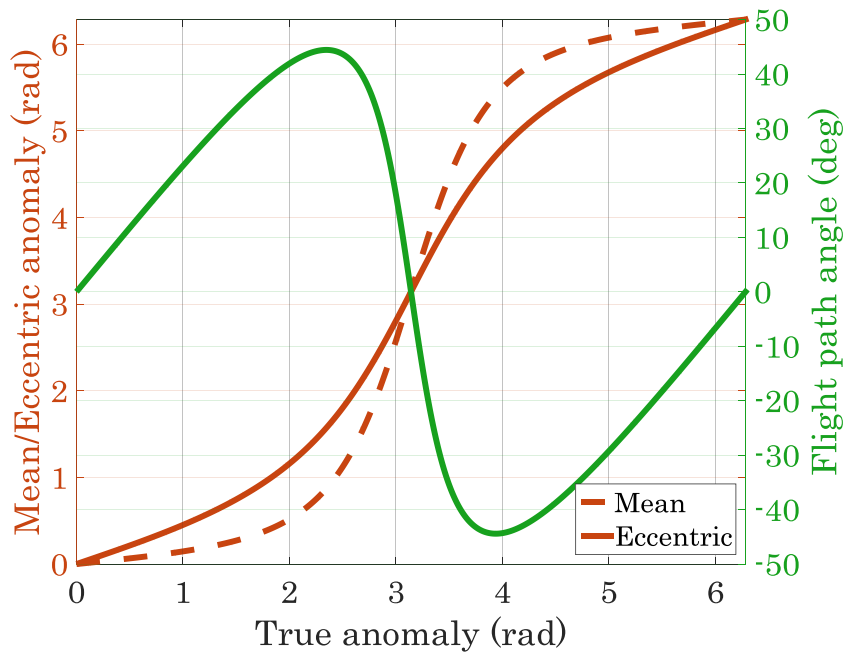

Figure 8: Variation of flight path angle, mean and eccentric anomalies $(e=0.7)$

\subsection{Ground Track}

Simulation of the ground track is one of the key elements in orbital mechanics, specifically for space mission design and tracking control [14]. It helps to generate appropriate track distance and revisit frequency over a given area. Different approaches in space engineering are based on ground track analysis, which permits the construction of ground track patterns and the determination of satellite arrangements. One sample representation is illustrated in Fig. 9 for a space orbit with semimajor axis of $28000 \mathrm{~km}$ and eccentricity of 0.3 , inclined by $60^{\circ}$.

In Fig. 9, the projection of a satellite's orbit onto the Earth's surface is plotted for 5 days of satellite motion. As can be seen, the satellite reaches a maximum and minimum amplitude during each revolution while passing over the equator twice, therefore on a mercator projection, the ground track of the satellite resembles a sine-like curve. Since the Earth rotates eastward beneath the satellite orbit at $15.04 \mathrm{deg} / \mathrm{h}$, the ground track advances westward at that rate.

Another family of orbits which is a matter of interest while examining the satellite

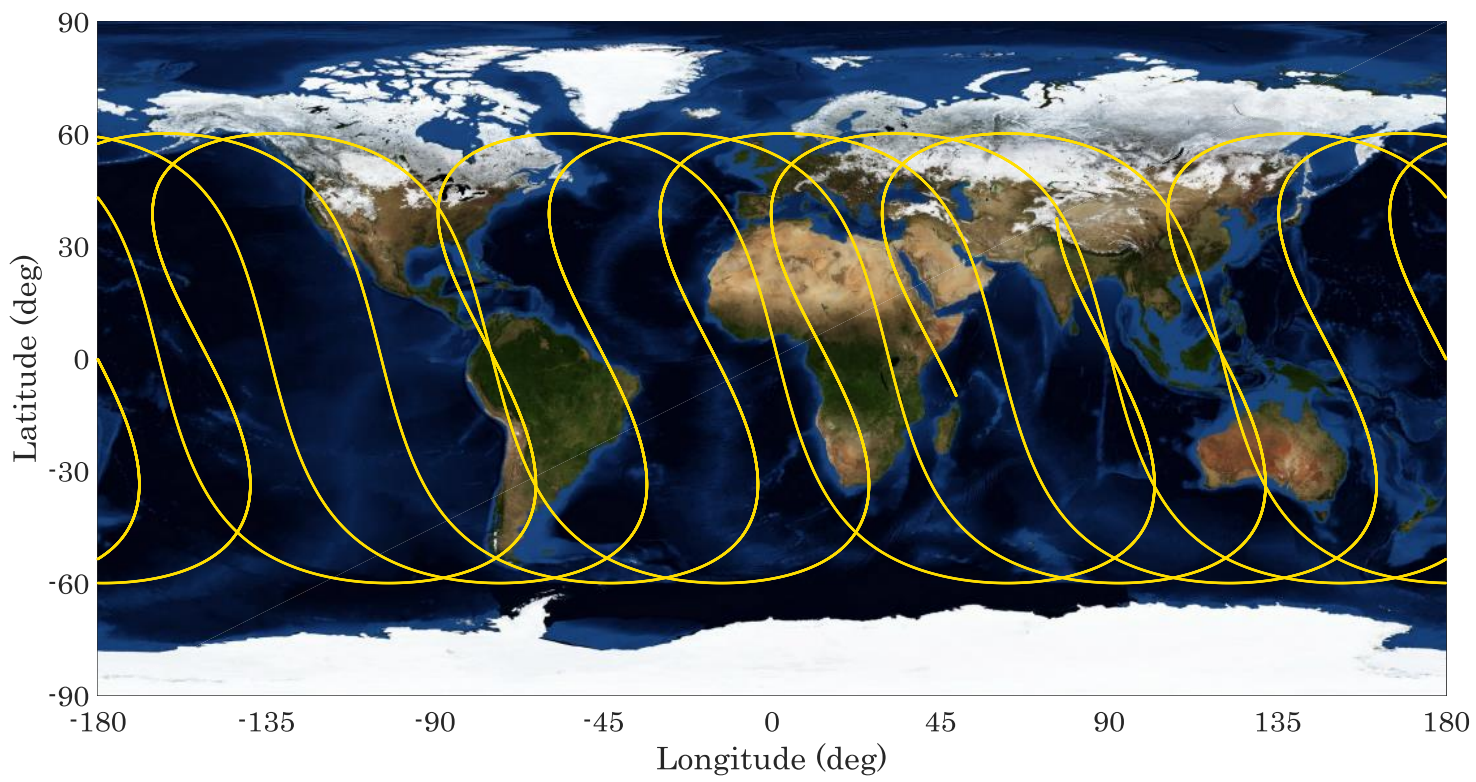

Figure 9: Satellite ground track $\left(a=28000 \mathrm{~km}, e=0.3, i=60^{\circ}, \Omega=30^{\circ}, \omega=190^{\circ}\right)$ 
ground tracks is the geosynchronous orbits. The elliptical geosynchronous orbits create drifts east and west as the spacecraft travels faster or slower at different points on its trajectory. Different combinations of inclination and eccentricity yields a motion relative to a fixed point on the ground track. Fig. 10 illustrates three instances of geosynchronous orbit ground tracks.

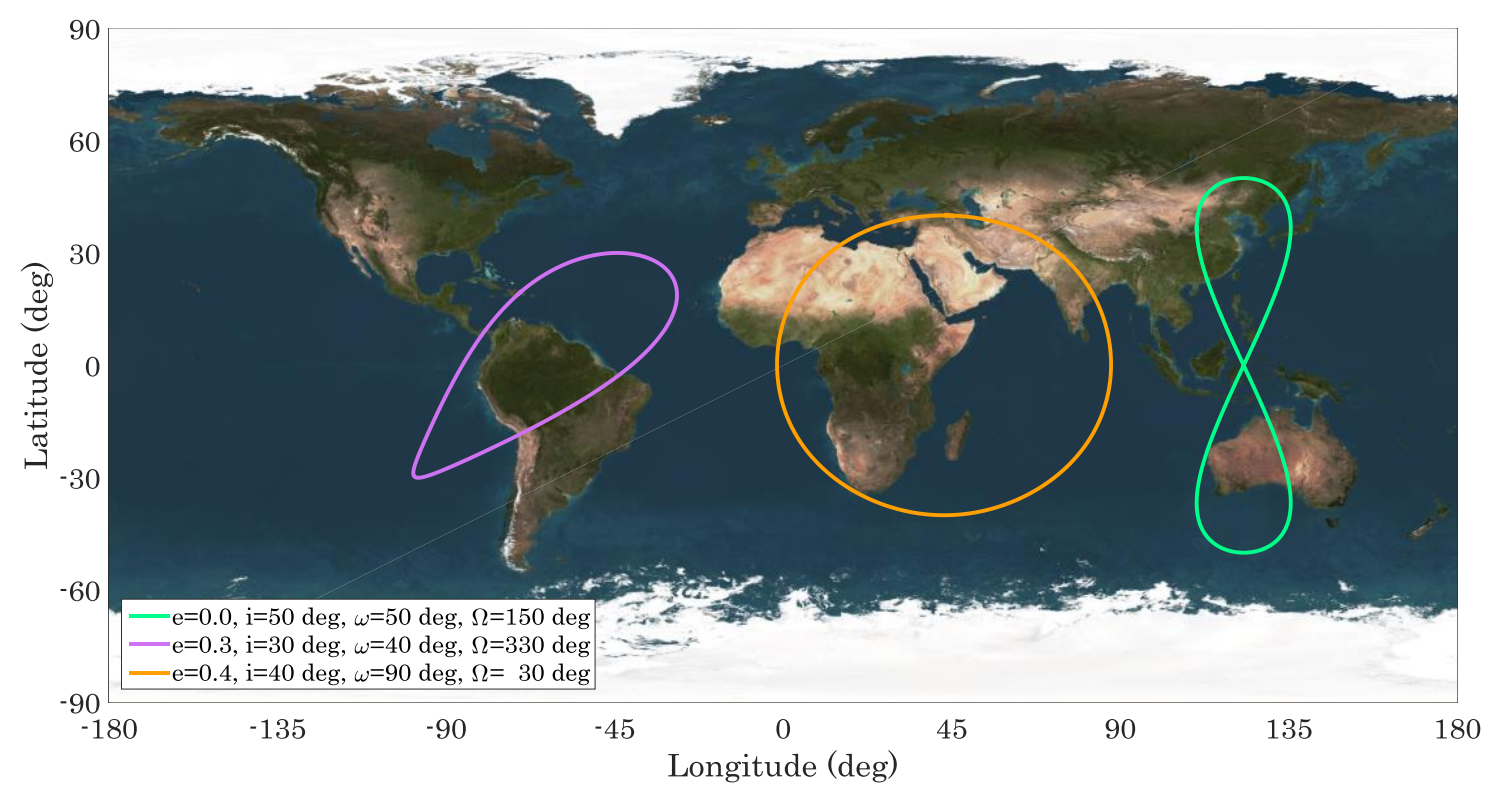

Figure 10: Geosynchronous ground tracks

The eight-like ground track (green line) is that of the geosynchronous orbit with no eccentricity. Obviously, changing the inclination of this orbit to zero makes this geosynchronous a geostationary orbit in which the satellite sits fixed at the crossover point of the eight-like shape (over the equator). On the other hand, if the eccentricity is increased, it results in a slanted teardrop shape. Typically, eccentric geosynchronous orbits will result in a slanted figure-eight. It just happens to have the crossover point at the northern apex of the ground track. Combining these modifications with various argument of perigees results different coverage areas for the geosynchronous orbits (orange and purple lines).

\subsection{Autonomous Space Rendezvous}

The presented framework is capable of linking with various optimization algorithms for solving spacecraft trajectory optimization problems such as interplanetary transfers [36] and space rendezvous [37]. In this section, a multi-impulse trajectory optimization problem is optimized and simulated to illustrate the capability of this tool. An evolutionary algorithm based on a discretized Lambert problem as described in [35] is linked to the platform to solve a long-range space rendezvous problem considering impulse limit.

In a rendezvous maneuver, two orbiting vehicles observe one another from each of their own free-falling, rotating, clearly non-inertial frames of reference. A rendezvous maneuver usually involves a target space vehicle, which is passive and non-maneuvering, and a chase spacecraft, which is active and performs the maneuvers required to bring itself alongside the target. In the long-range space rendezvous, the chaser executes several maneuvers under the guidance of the ground telemetry tracking and command network, so that the navigation sensors of the chaser can catch the target. The major objectives of this phase include adjusting the phase angle between the two spacecraft, reducing the orbital plane differences, increasing the orbital height, and initiating the relative navigation. The initial states of the chaser and the target spacecraft for the selected mission are provided in Table 1. 
Table 1: Initial states of two spacecraft in long-range space rendezvous

\begin{tabular}{lrr}
\hline Orbital elements & Chaser & Target \\
\hline$a(\mathrm{~km})$ & 7000 & 30000 \\
$e$ & 0.0 & 0.1 \\
$i(\mathrm{deg})$ & 60 & 0 \\
$\Omega(\mathrm{deg})$ & 0 & 80 \\
$\omega(\mathrm{deg})$ & 205 & 225 \\
$\theta(\mathrm{deg})$ & 3 & 120 \\
\hline
\end{tabular}

The optimal sequence of impulses is obtained and transfer trajectories are simulated within the proposed framework. 3D illustration of the transfer trajectories are depicted in Fig. 11.

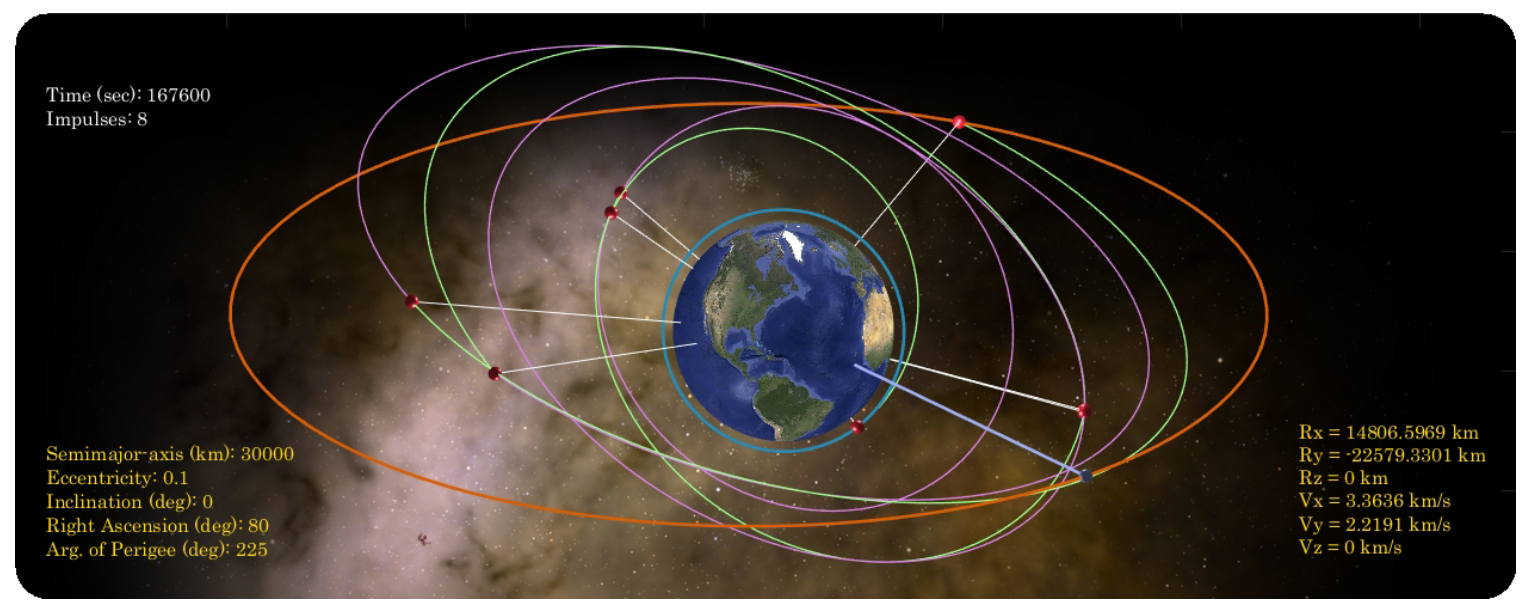

Figure 11: 3D visualization of multi-impulse long-range space rendezvous

In this figure, the initial and final orbits are illustrated along with coasting trajectories between the impulses during the space mission. In this scenario, eight impulses fulfill the orbital maneuver with respect to the mission criteria. The location and the corresponding radius of the impulses are shown in the figure, indicating the anomalies of the space vehicle at the intersection of transfer trajectories. Details regarding the variation of orbital elements due to the act of impulses are shown in Fig. 12.

As it is shown, the changes of five orbital elements, including semi-major axis $(a)$, eccentricity $(e)$, inclination $(i)$, right ascension of ascending node $(\Omega)$, argument of perigee $(\omega)$, along with true anomaly indicates the time of the impulses acted on the chaser spacecraft. The fluctuations within the plots are due to the fact that when the impulse is acted on the chaser, the orbital plane and the shape of the orbit is changed, placing the chaser on a new trajectory during the process of reaching the target space vehicle. These variations correspond with the changes of the state vectors of the chaser, including the position $(\vec{r})$ and the velocity $(\vec{v})$ of the vehicle as shown in Fig. 13 and Fig. 14. Similarly, non-continuity in the velocity components are due to the fact that the maneuvers are impulsive, not with continuous thrust.

As the figures indicate, the state vectors of the chaser converge to the states of the target as the spacecraft travels on the coasting trajectories. According to the simulation results, the time between two sequential impulses, which increases as the chaser approaches the target is increasing. It agrees with the fact that since the orbital period of the target orbit is more than the initial orbit, the chaser transfers to orbits with higher angular velocities. Fig. 15 indicates the location of the impulses on the map.

In this figure, the ground track of the chaser within the orbital maneuver is also illustrated. The size of the red circles corresponds to the magnitude of impulses in each location. 

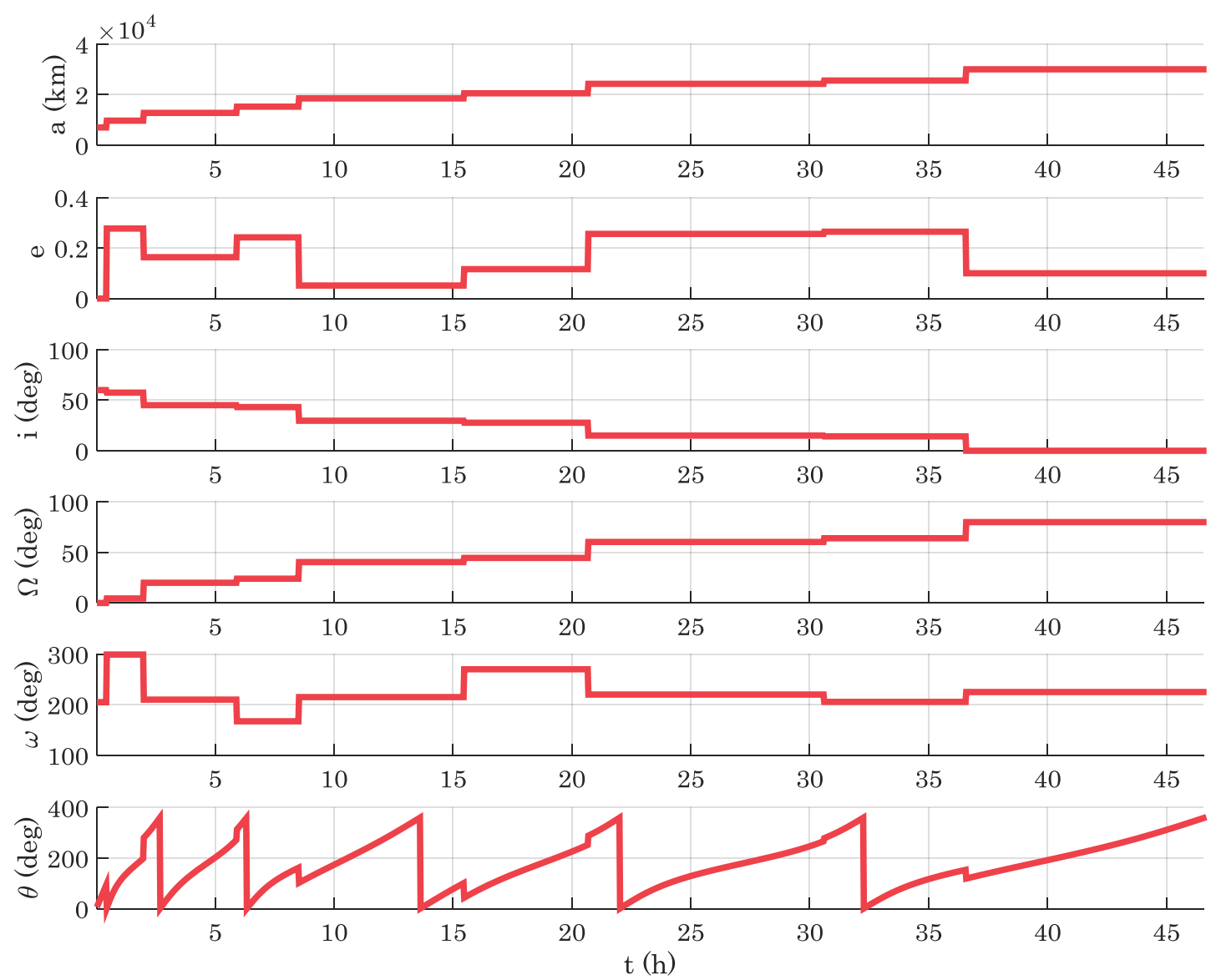

Figure 12: Variation of orbital elements of the chaser spacecraft
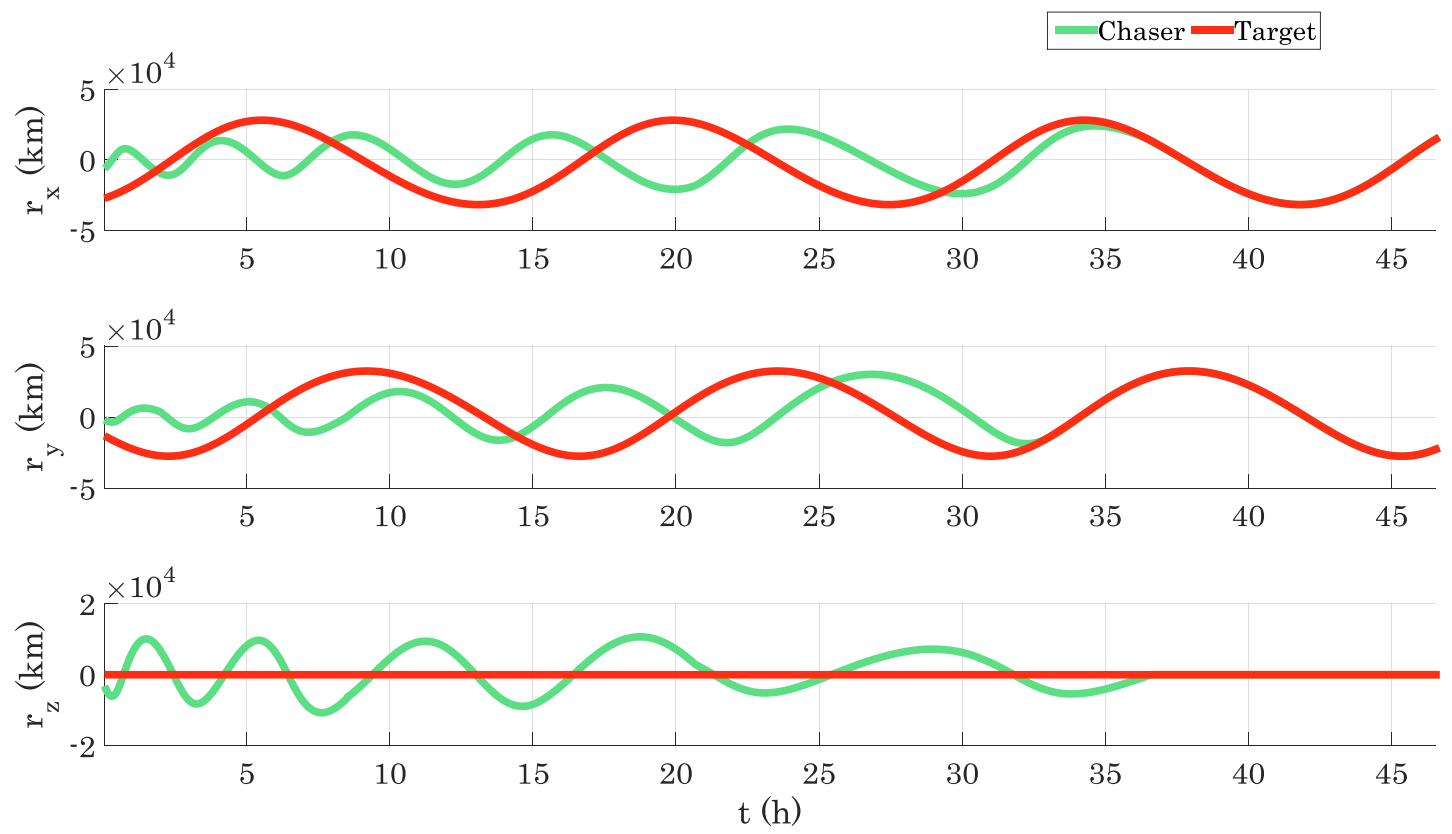

Figure 13: Position states of the chaser and the target spacecraft

Each impulse is acted on the chaser in a different altitude, as detailed in Table 2.

According to the results, the chaser starts its first transfer in its initial trajectory, which is an inclined orbit. As the maneuver proceeds, the impulses occur at higher altitudes, which makes the distance between target and chaser decrease. After the final impulse, the 

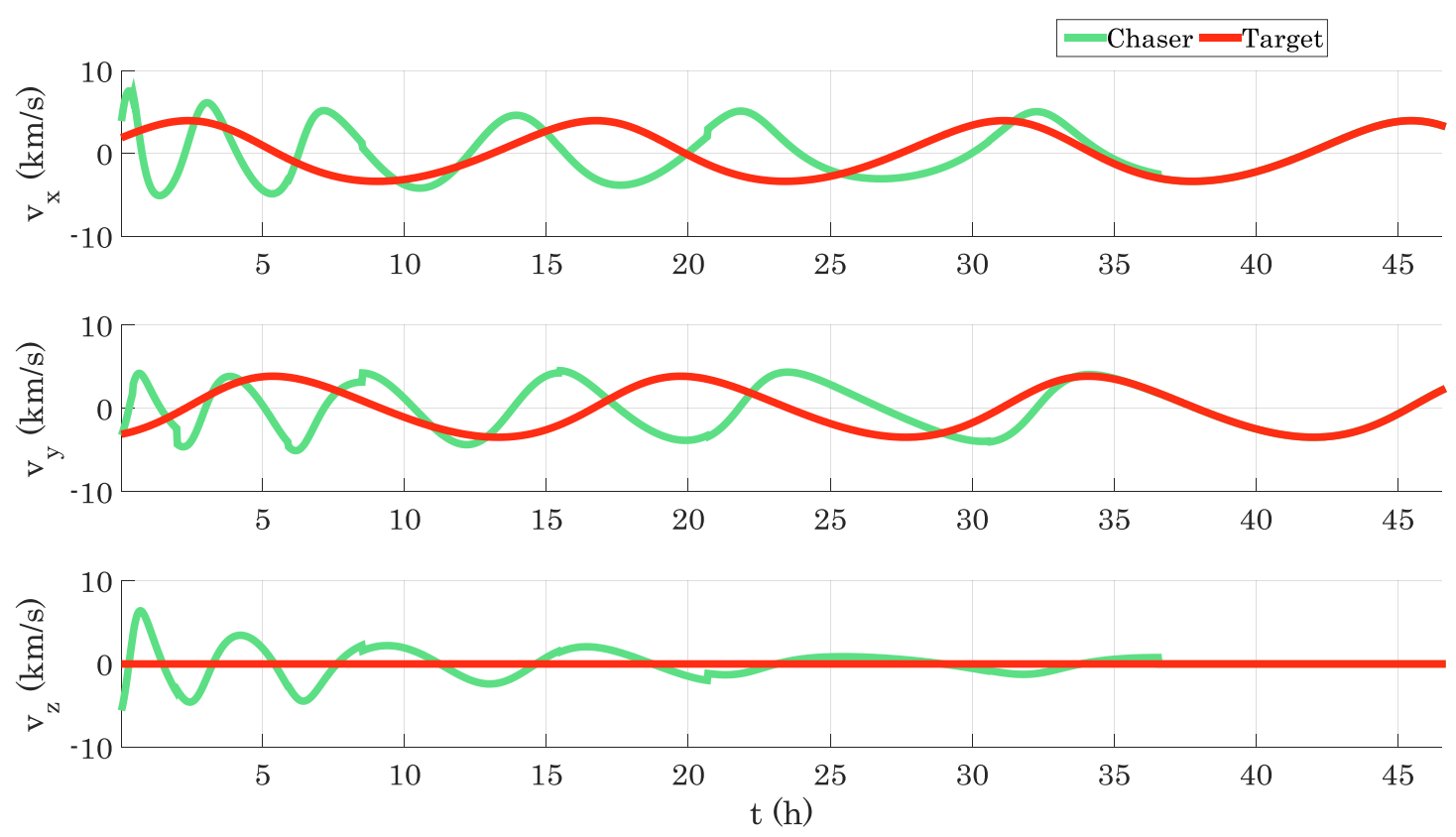

Figure 14: Velocity states of the chaser and the target spacecraft

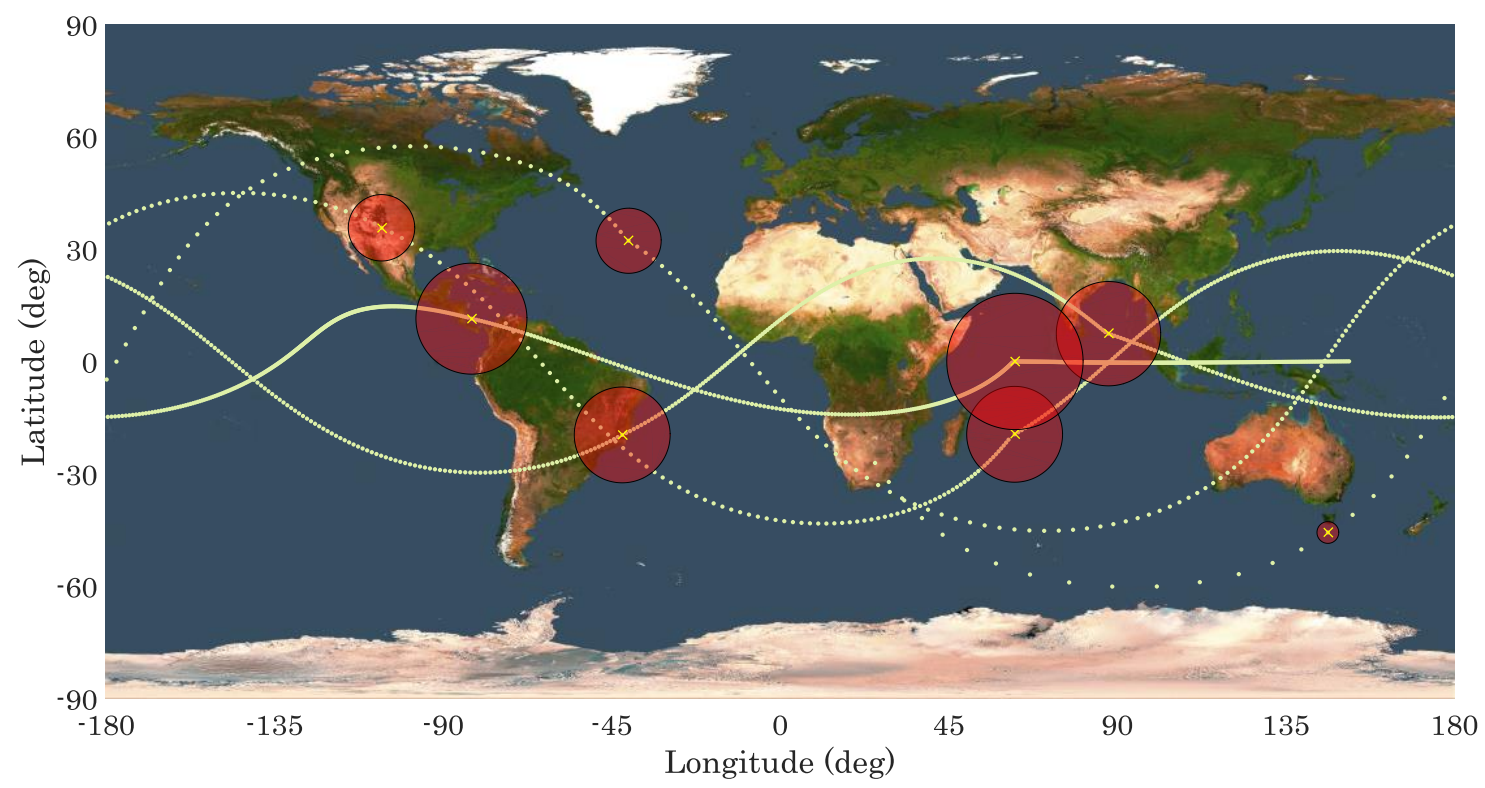

Figure 15: Ground track of the chaser and the location of impulses

Table 2: Geographic coordinates of the impulses acted on the chaser

\begin{tabular}{lllll}
\hline Impulse & Time $(\mathrm{s})$ & Latitude $(\mathrm{deg})$ & Longitude $(\mathrm{deg})$ & Altitude $(\mathrm{km})$ \\
\hline 1 & 1600 & -45.5765 & 146.2579 & 632.7572689 \\
2 & 7200 & 32.33925 & -40.4453 & 5670.861950 \\
3 & 21300 & 35.80216 & -106.418 & 5899.997229 \\
4 & 30700 & -19.3534 & 62.62282 & 12282.44339 \\
5 & 55700 & -19.5134 & -42.1559 & 12285.10654 \\
6 & 74500 & 7.51914 & 87.68698 & 14559.53319 \\
7 & 110200 & 11.53168 & -82.4076 & 16475.49403 \\
8 & 131700 & 0.105248 & 62.70357 & 24837.11411 \\
\hline
\end{tabular}


chaser is at the same position and velocity of the target where the orbit inclination becomes zero.

\subsection{Results Validation}

Several verification tests are performed for validating the simulation results in HOMA. The orbit propagation process is the main subject which has been verified through different comparisons. The two perturbed orbit propagation models, SGP4 and HPOP are considered for validation. Results are compared with the outputs of two similar simulation platforms, STK by Analytical Graphics, Inc. and PROPAT by Carrara. While the SGP4 propagation scheme is tested with the former toolbox, the HPOP propagation model is compared with the latter framework.

The two-line element set in Table 3 is considered as the input for SGP4 propagation in STK as well as in HOMA. The TLE is given for IRIDIUM 162, retrieved from CelesTrak [38]. The satellite is a part of the Iridium constellation, launched in May 2018. The simulation results are illustrated in Fig. 16 to 21. Each figure shows the variation of one orbital elements and the variations are depicted for HOMA and STK respectively. Also, the maximum value of absolute error, (E), for each quantity is extracted from the figures.

Since the SGP4 model is used here, the orbital elements are not constant as the time passes. According to the comparison, the overall shape of the outputs are the same, leading

Table 3: IRIDIUM 162 two-line element (June 26th 2018)

\begin{tabular}{l|l}
1 & $43482 \mathrm{U} 18047 \mathrm{G} 18177.64341180+.00000099+00000-0+23569-409999$ \\
\hline 2 & 43482086.4507225 .56080002381102 .6517257 .495014 .43275512005031
\end{tabular}

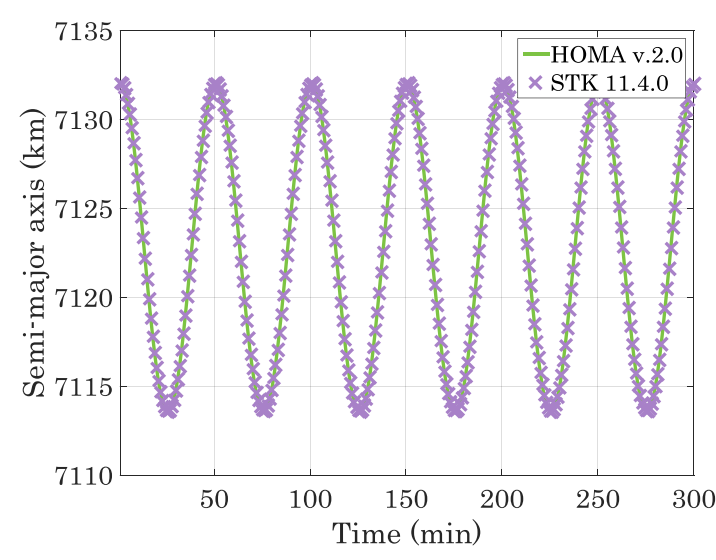

Figure 16: Semi-major axis $\left(E_{a}=6.4 m\right)$

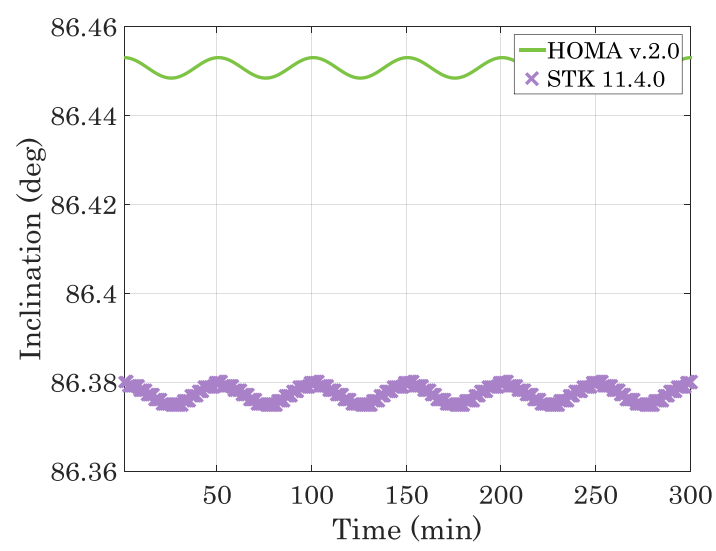

Figure 18: Inclination $\left(E_{i}=0.073^{\circ}\right)$

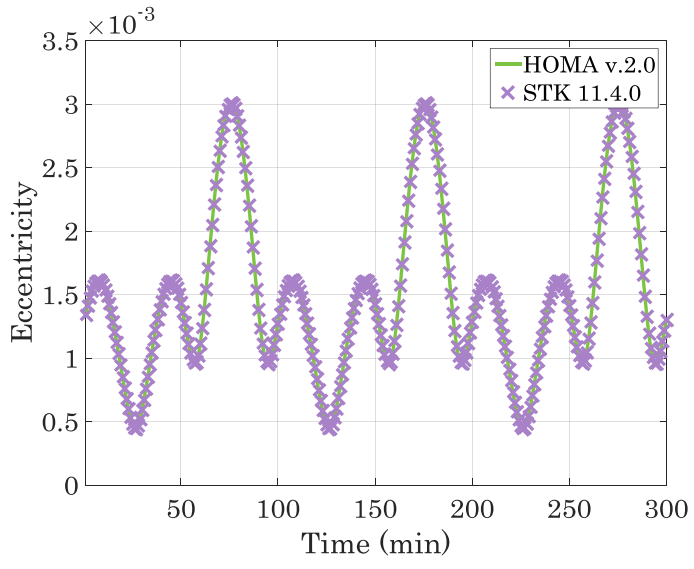

Figure 17: Eccentricity $\left(E_{e}=1.38 e-6\right)$

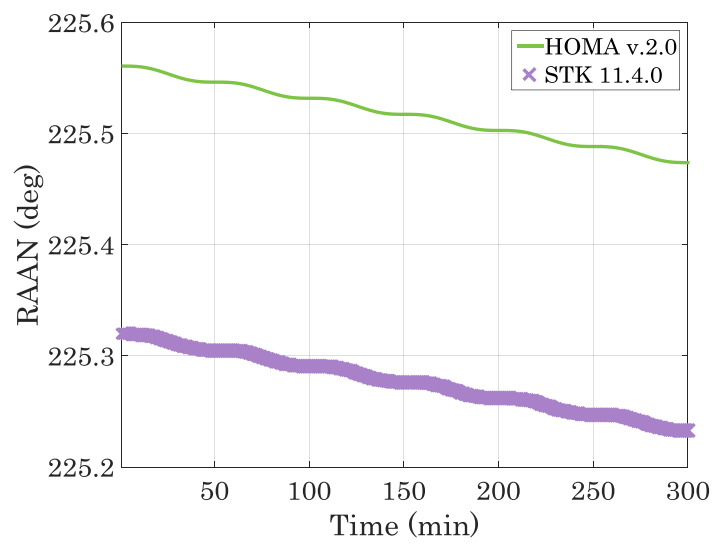

Figure 19: $\operatorname{RAAN}\left(E_{\Omega}=0.241^{\circ}\right)$ 


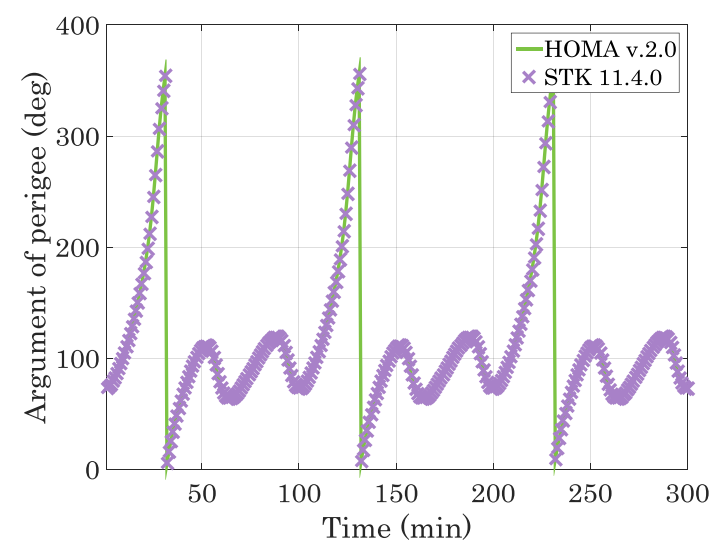

Figure 20: Arg. of perigee $\left(E_{\omega}=0.142^{\circ}\right)$

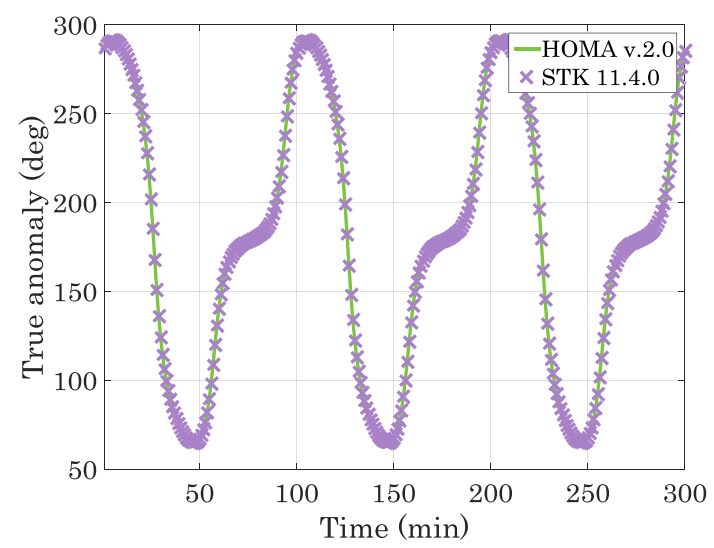

Figure 21: True anomaly $\left(E_{\theta}=0.072^{\circ}\right)$

to conclude that the perturbed trajectory is formulated properly regarding the SGP4 orbit propagation model. The differences are also negligible regarding the type of each element, and acceptable for preliminary space orbit simulation and design as in typical space missions.

The results regarding the high precision orbit propagation are compared with the Brower model in PROPAT, in which the gravitational field of the Earth along with the flattening of the poles are considered. Considering an elliptical orbit with semi-major axis of $19000 \mathrm{~km}$ and eccentricity of 0.45 , inclined by $65^{\circ}$ with right ascension of ascending node of $5^{\circ}$ and argument of perigee of $25^{\circ}$. The state vectors of the satellite at perigee is $\vec{r}=\left[\begin{array}{lll}9489.07 & 2507.12 & 3598.32\end{array}\right] \mathrm{km}$ and $\vec{v}=\left[\begin{array}{lll}-3.16 & 2.62 & 6.19\end{array}\right] \mathrm{km} / \mathrm{s}$ in the ECI frame. Propagation of space orbit is done within 10 uniform time steps. The relative percentage errors $(\hat{E})$ for state variables with respect to PROPAT results are tabulated in Table 4.

As can be seen, the maximum value of relative percentage error is within the order of $10^{-2}$ after one period. Note that the orbital period of the selected orbit is 26064 seconds.

\section{Conclusion and Remarks}

Simulation in space engineering is essential for autonomous spacecraft mission design and analysis. Taking advantage of graphical user interfaces, HOMA generates numerical data of space orbit simulation in a 3D visual representation that makes the trajectory design and optimization process much easier. It is incorporated with a framework that gives the scientists in astrodynamics and space engineers the ability to achieve accurate results from the simulations.

This paper presents the on-going research work on developing a high-fidelity gravitational simulator as applied to orbital mechanics and mission trajectory design. Given the

Table 4: Relative percentage error of state vectors

\begin{tabular}{rcccccc}
\hline Time $(\mathrm{s})$ & $\hat{E}_{r_{x}}$ & $\hat{E}_{r_{y}}$ & $\hat{E}_{r_{z}}$ & $\hat{E}_{v_{x}}$ & $\hat{E}_{v_{y}}$ & $\hat{E}_{v_{z}}$ \\
\hline 2600 & $2.19 \mathrm{E}-04$ & $3.56 \mathrm{E}-04$ & $1.13 \mathrm{E}-04$ & $1.92 \mathrm{E}-04$ & $1.24 \mathrm{E}-02$ & $3.96 \mathrm{E}-04$ \\
5200 & $8.55 \mathrm{E}-05$ & $1.22 \mathrm{E}-03$ & $2.56 \mathrm{E}-04$ & $1.76 \mathrm{E}-04$ & $5.65 \mathrm{E}-04$ & $3.39 \mathrm{E}-04$ \\
7800 & $1.54 \mathrm{E}-04$ & $1.32 \mathrm{E}-02$ & $1.35 \mathrm{E}-03$ & $9.07 \mathrm{E}-04$ & $1.54 \mathrm{E}-05$ & $3.27 \mathrm{E}-04$ \\
10400 & $3.60 \mathrm{E}-04$ & $1.54 \mathrm{E}-04$ & $2.45 \mathrm{E}-03$ & $1.51 \mathrm{E}-02$ & $4.35 \mathrm{E}-05$ & $1.82 \mathrm{E}-04$ \\
13000 & $7.57 \mathrm{E}-04$ & $2.00 \mathrm{E}-04$ & $8.74 \mathrm{E}-04$ & $3.46 \mathrm{E}-03$ & $3.71 \mathrm{E}-04$ & $1.95 \mathrm{E}-04$ \\
15600 & $1.55 \mathrm{E}-03$ & $1.34 \mathrm{E}-04$ & $3.87 \mathrm{E}-04$ & $2.14 \mathrm{E}-03$ & $2.22 \mathrm{E}-03$ & $1.27 \mathrm{E}-03$ \\
18200 & $3.56 \mathrm{E}-03$ & $3.05 \mathrm{E}-04$ & $1.70 \mathrm{E}-04$ & $1.61 \mathrm{E}-03$ & $7.14 \mathrm{E}-02$ & $7.60 \mathrm{E}-03$ \\
20800 & $1.75 \mathrm{E}-02$ & $1.57 \mathrm{E}-03$ & $1.31 \mathrm{E}-03$ & $8.14 \mathrm{E}-04$ & $7.68 \mathrm{E}-03$ & $1.20 \mathrm{E}-02$ \\
23400 & $8.17 \mathrm{E}-03$ & $6.80 \mathrm{E}-03$ & $5.46 \mathrm{E}-03$ & $3.36 \mathrm{E}-03$ & $4.82 \mathrm{E}-03$ & $5.94 \mathrm{E}-03$ \\
26000 & $3.96 \mathrm{E}-03$ & $2.74 \mathrm{E}-02$ & $5.78 \mathrm{E}-02$ & $2.66 \mathrm{E}-02$ & $3.83 \mathrm{E}-03$ & $1.79 \mathrm{E}-03$ \\
\hline
\end{tabular}


widespread use of MATLAB from The Mathworks Inc. in space engineering, as well as the inherent advantages of using different packages with powerful mathematical functions and display characteristics, a toolbox for simulating space orbits was developed under this platform.

This toolbox is aimed at developing a customizable application which addresses the mission needs of satellite orbit simulations, and which analyzes scenarios considering all the perturbations influencing the orbit propagation. The results were assessed against simulations of commercially available simulation tools and were found to match satisfactorily.

Indeed, the simulation platform described in this paper can be expanded to a wider range of designs and analyses of space orbits, such as libration points, trajectory optimization, and the requirements to reach a specified orbit with the minimum amount of fuel. Given the flexibility of this application, it can be extended to perform extensive studies in orbital mechanics. Future research can also be dedicated to the development of a platform for the comparison of propagation models in terms of accuracy and computational time.

\section{Acknowledgments}

This research is supported by La Caixa Foundation Fellow-ship, the Basque Government through the BERC 2018-2021, Elkartek programs, Spanish Ministry of Economy and Competitiveness MINECO: BCAM Severo Ochoa excellence accreditation SEV-2017-0718, TIN2016-78365R and TIN2017-82626R projects, Spanish Ministry of Science PID20191064536A-I00, Basque Government consolidated groups 2019-2021 IT1244-19.

\section{References}

[1] L. Feruglio, S. Corpino, Neural networks to increase the autonomy of interplanetary nanosatellite missions, Robotics and Autonomous Systems 93 (2017) 52-60.

[2] B. Davoudi, E. Taheri, K. Duraisamy, B. Jayaraman, I. Kolmanovsky, Quad-rotor flight simulation in realistic atmospheric conditions, arXiv preprint arXiv:1902.01465.

[3] S. L. May, B. Carter, S. Gehly, S. Flegel, M. Jah, Representing and querying space object registration data using graph databases, Acta Astronautica 173 (2020) 392-403.

[4] B. Jones, E. Delande, E. Zucchelli, M. Jah, Multi-fidelity orbit uncertainty propagation with systematic errors, amos (2019) 14.

[5] A. Shirazi, J. Ceberio, J. A. Lozano, Spacecraft trajectory optimization: A review of models, objectives, approaches and solutions, Progress in Aerospace Sciences 102 (2018) 76-98.

[6] J. A. Dominguez, E. Victor, A. R. Urbina, A. L. Vasquez, Comprehensive software simulation on ground power supply for launch pads and processing facilities at NASA kennedy space center, IEEE Aerospace and Electronic Systems Magazine 34 (8) (2019) $4-11$.

[7] A. Graphics, Incorporated (agi). stk user's manual [k]. version5. 0.1. extort, pa, usa: Analytical graphics, Inc (AGI).

[8] D. J. Conway, S. P. Hughes, The general mission analysis tool (gmat): Current features and adding custom functionality, in: 4th International Conference on Astrodynamical Tools and Techniques, 2010, pp. 1-8.

[9] V. Carrara, An open source satellite attitude and orbit simulator toolbox for matlab, in: Proceedings of the 17th International Symposium on Dynamic Problems of Mechanics, 2015, pp. 1-12. 
[10] D. Brouwer, G. M. Clemence, Methods of celestial mechanics, Academic, New York, 1961.

[11] S. Habibkhah, J. Arasi, H. Bolandi, SPACSSIM: Simulation and analysis software for mathematical modeling of satellite position and attitude control system, Computing in Science \& Engineering (2017) 1-1.

[12] O. R. Fernandez, J. Utzmann, U. Hugentobler, SPOOK - a comprehensive space surveillance and tracking analysis tool, Acta Astronautica.

[13] A. Shirazi, HOMA - Online Space Orbit Simulator, accessed: 2020-12-14 (2014). URL http://www.homasim.com/

[14] D. A. Vallado, Fundamentals of Astrodynamics and Applications, 4th Ed., Microcosm Press, 2013.

[15] R. A. Broucke, P. J. Cefola, On the equinoctial orbit elements, Celestial Mechanics 5 (3) (1972) 303-310.

[16] D. A. Vallado, P. J. Cefola, Two-line element sets-practice and use, in: 63rd International Astronautical Congress, Naples, Italy, 2012, pp. 1-14.

[17] W. E. Wiesel (Ed.), Space Flight Dynamics, CreateSpace Independent Publishing Platform, 2010.

[18] H. D. Curtis, Orbital mechanics for engineering students, Butterworth-Heinemann, 2014.

[19] F. R. Hoots, R. L. Roehrich, Models for propagation of norad element sets, Tech. rep., Aerospace defense command Peterson AFB Co-office of Astrodynamics (1980).

[20] D. Brouwer, Solution of the problem of artificial satellite theory without drag, The Astronomical Journal 64 (1959) 378.

[21] Y. Kozai, The motion of a close earth satellite, The Astronomical Journal 64 (1959) 367.

[22] M. Lane, P. Fitzpatrick, J. Murphy, On the representation of air density in satellite deceleration equations by power functions with integral exponents, Tech. rep., Air Proving Ground Center EGLIN AFB FL (1962).

[23] D. Vallado, P. Crawford, Sgp4 orbit determination, in: AIAA/AAS Astrodynamics Specialist Conference and Exhibit, 2008, p. 6770.

[24] W. M. Kaula, Theory of satellite geodesy: applications of satellites to geodesy, Courier Corporation, 2013.

[25] J. M. Wahr, Body tides on an elliptical, rotating, elastic and oceanless earth, Geophysical Journal International 64 (3) (1981) 677-703.

[26] R. Eanes, Earth and ocean tide effects on lageos and starlette, in: Proceedings of the Ninth International Symposium on Earth Tides, E. Sckweizerbart'sche Verlagabuchhandlung, 1983, pp. 1-17.

[27] K. Lambeck, The Earth's variable rotation: geophysical causes and consequences, Cambridge University Press, 2005.

[28] E. Standish, J. Planetary, L. Ephemerides, De405/le405, memo iom 312, Tech. rep., F-98-048 (1998). 
[29] C. Huang, J. Ries, B. Tapley, M. Watkins, Relativistic effects for near-earth satellite orbit determination, Celestial Mechanics and Dynamical Astronomy 48 (2) (1990) 167185.

[30] J. Ries, C. Huang, M. Watkins, Effect of general relativity on a near-earth satellite in the geocentric and barycentric reference frames, Physical review letters 61 (8) (1988) 903.

[31] B. Schutz, B. Tapley, Orbit accuracy assessment for seasat, Journal of the Astronautical Sciences 28 (1980) 371-390.

[32] B. Tapley, J. Ries, Orbit determination requirements for topex, in: Astrodynamics 1987, 1988, pp. 321-338.

[33] P. Knocke, J. Ries, B. Tapley, Earth radiation pressure effects on satellites, in: Astrodynamics Conference, 1988, p. 4292.

[34] H. Fliegel, T. Gallini, E. Swift, Global positioning system radiation force model for geodetic applications, Journal of Geophysical Research: Solid Earth 97 (B1) (1992) $559-568$.

[35] A. Shirazi, J. Ceberio, J. A. Lozano, An evolutionary discretized lambert approach for optimal long-range rendezvous considering impulse limit, Aerospace Science and Technology (2019) 105400.

[36] M. Saghamanesh, E. Taheri, H. Baoyin, Systematic low-thrust trajectory design to mars based on a full ephemeris modeling, Advances in Space Research.

[37] A. Shakouri, M. Kiani, S. H. Pourtakdoust, Covariance-based multiple-impulse rendezvous design, IEEE Transactions on Aerospace and Electronic Systems 55 (5) (2019) $2128-2137$.

[38] T. Kelso, CelesTrak - Public Domain Satellite Tracking Data, accessed: 2020-12-14 (2010).

URL http://celestrak.com/ 